\title{
BUDDHIST STONE SCRIPTURES FROM SHANDONG, CHINA
}

\author{
Matthias Arnold \\ Heidelberg Academy of Sciences and Humanities \\ Research Group Buddhist Stone Scriptures in China \\ Hauptstraße 113 \\ 69117 Heidelberg \\ Germany \\ marnold@zo.uni-heidelberg.de \\ http://www.stonesutras.org
}

\begin{abstract}
This paper introduces the website of the Buddhist Stone Scriptures project of the Heidelberg Academy of Sciences. Based on the TEI guidelines we store textual and visual data together with GIS information in a XML database. To access the inscriptions we provide visual tools like zoomable maps or Flashinterfaces as means of navigation. A special problem is the display of a single inscription, as multiple layers of information in combination with heavily annotated text in a critical apparatus need to be displayed on a single page. The project developed the "Five Layers" model that is discussed in more detail.
\end{abstract}

\section{INTRODUCTION}

In the second half of the 6th century Buddhist monks began in an area which corresponds to present day Shandong Province to engrave passages from sutra texts onto stone. They carved the characters on the bare rock under the open sky. About 30 such sites exist. Some contain only a few characters, but the largest one, the Diamond sutra on Mount Tai, had originally about 2,500 characters of which ca. 1,200 are still extant.

These monuments, embedded into the landscape, are among the most impressive testimonies of religious art in China. They also mark a high point in the history of Chinese calligraphy. As a kind of land art which turns the environment into a holy realm by aesthetic means they are unique in the world.

The project Buddhist Stone Scriptures of Shandong Province was launched in 2005 by the Ji'nan Museum for the Art of Stone Engravings of Shandong province and the Heidelberg Academy of Sciences and Humanities. For the documentation and interpretation, the project relies on international cooperation, notably with scholars from China and Japan. For the presentation, the project has prepared a website based on an XML database system and uses GIS data and maps as well as 3D models and video sequences for each inscription site. 


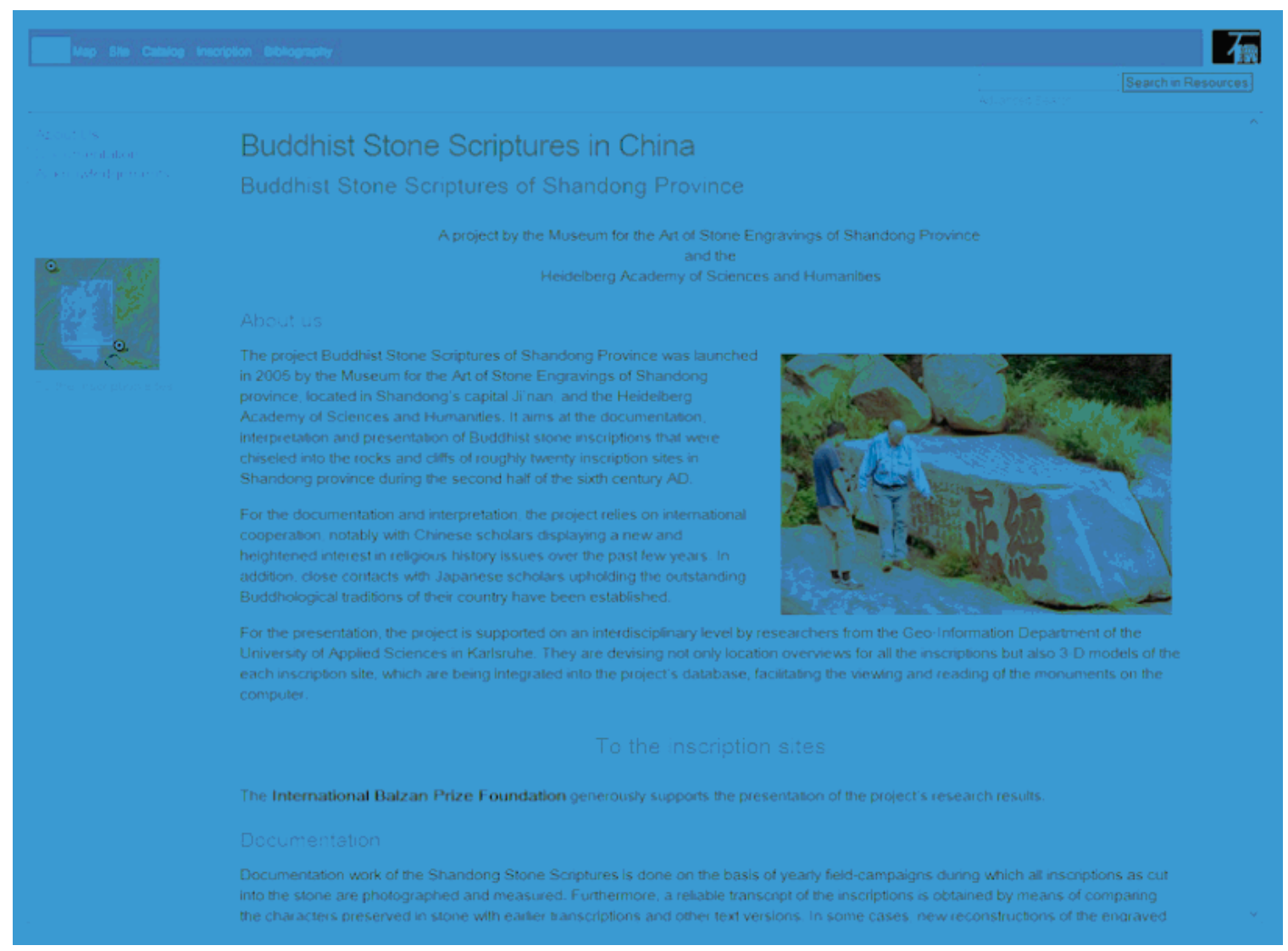

Figure 1 - Main page of the website

\section{The data}

The project works with four different types of data: All inscriptions are photographed in situ and photographs are also taken of rubbings. In addition, photographs documenting the fieldwork and short video sequences like virtual flights. The texts are analyzed, transcribed into modern Chinese and translated into English. Together with a full critical apparatus including annotations, comments, and references this material forms the textual data. A special type is what we call "Catalog", comprising core metadata information on site and inscription. The third type of data is the geographical information, namely Geo-references of sites, inscriptions, and characters, detailed measurements, and maps. Finally, we incorporate or link to external resources, like passages from text corpora or dictionaries [1].

\section{The database}

All data is entered into a database using XML [2] with the TEI Guidelines for Electronic Text Encoding and Interchange [3] as the main standard. These guidelines provide a powerful markup language for texts and offer a rich set of advanced features, including elements for a critical apparatus or the representation of non-standard characters and glyphs as required in our project. We use TEI in combination with other XML 
schema languages, like MODS (Metadata Object Description Schema) [4] for bibliographic metadata and MADS (Metadata Authority Description Schema) [5] to record existing variants for personal and corporate names.

For storing the data we use the open source native XML database eXist [6] together with a number of other XML standards like XQuery, XPath or XSLT for processing it. The editing of our XML documents is either done directly in the database via a WebDAV enabled editor (i.e. Oxygen [7]), or we use online forms to enter data via web-interfaces, for example to annotate images (xPhoto tool), or to enter basic metadata for sites and inscriptions (Catalogue).

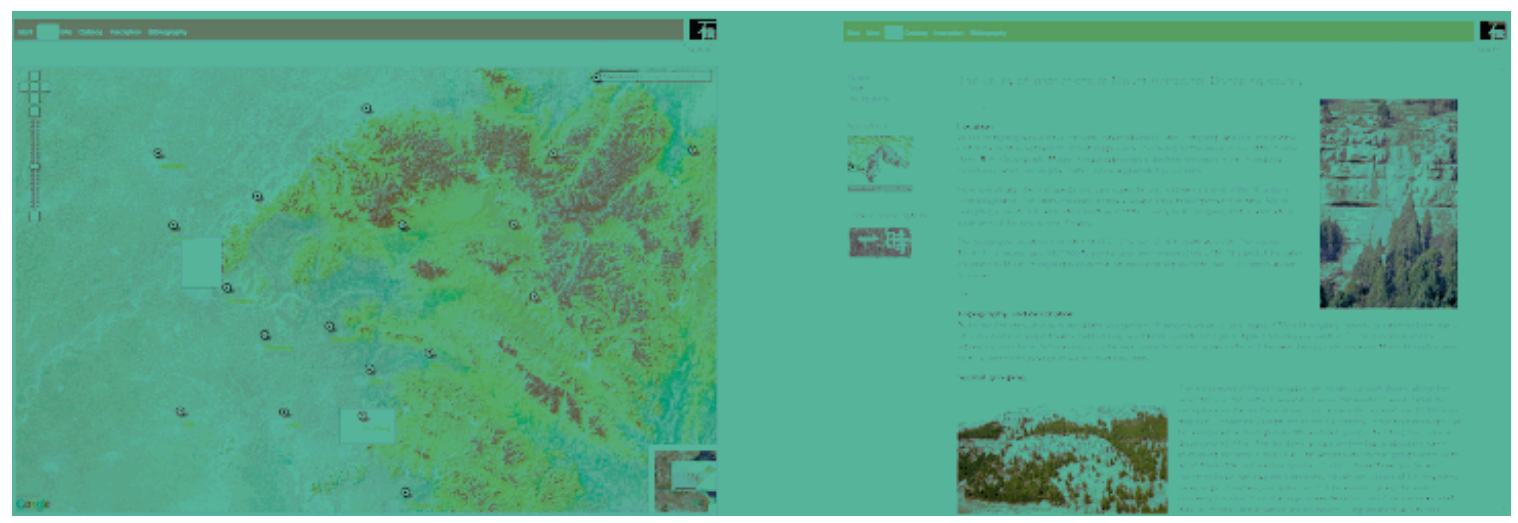

Figure 2. Map interface

Figure 3. Site: Mount Hongding

\section{The presentation}

The online presentation of the project is at http://stonesutras.org [8]. Its entire web interface is powered by XQuery and related XML- and Web-Standards, like XSLT, XHTML, CSS and JavaScript, and uses AJAX and other so-called Web 2.0 technologies. The website is designed for easy orientation within the project and to offer simple ways to access the sites and inscriptions. Users can access the inscriptions by either using the maps, by choosing a specific site from a pull-down list, or by using search function.

\section{Map}

The project has established a co-operation with researchers of the Geo-Information Department at the University of Applied Sciences in Karlsruhe. As one of the results, geo-references to the global coordinate system for all inscriptions are stored in the database. We have set up a GeoServer [9] and place our own Shandong province map on top of the satellite imagery provided by Google [10]. The interactive map with Google Earth functionality includes markers of sites and cities that are generated from our XML database. On our website the map functionality is provided as main tool to navigate between sites.

\section{Site}


Each site is introduced in terms of space, time and inscriptions. The section on "space" defines the location of the site, describes its characteristic topography, the grouping of inscriptions and elucidates on the place name. In the "time" section a site is placed in the context of Buddhist history and history in general, discussing its dating, or its relations to monasteries and historic figures. In the section on "inscriptions" the inscribed texts are divided in groups based on content and shape. They are positioned within the Buddhist transmission, compared with other versions, and conclusions are drawn upon text history. Finally, they are analyzed with focus on art historical characteristics, like engraving techniques and style of calligraphy. This scientific discussion is supplemented by images, video sequences and sometimes animations.

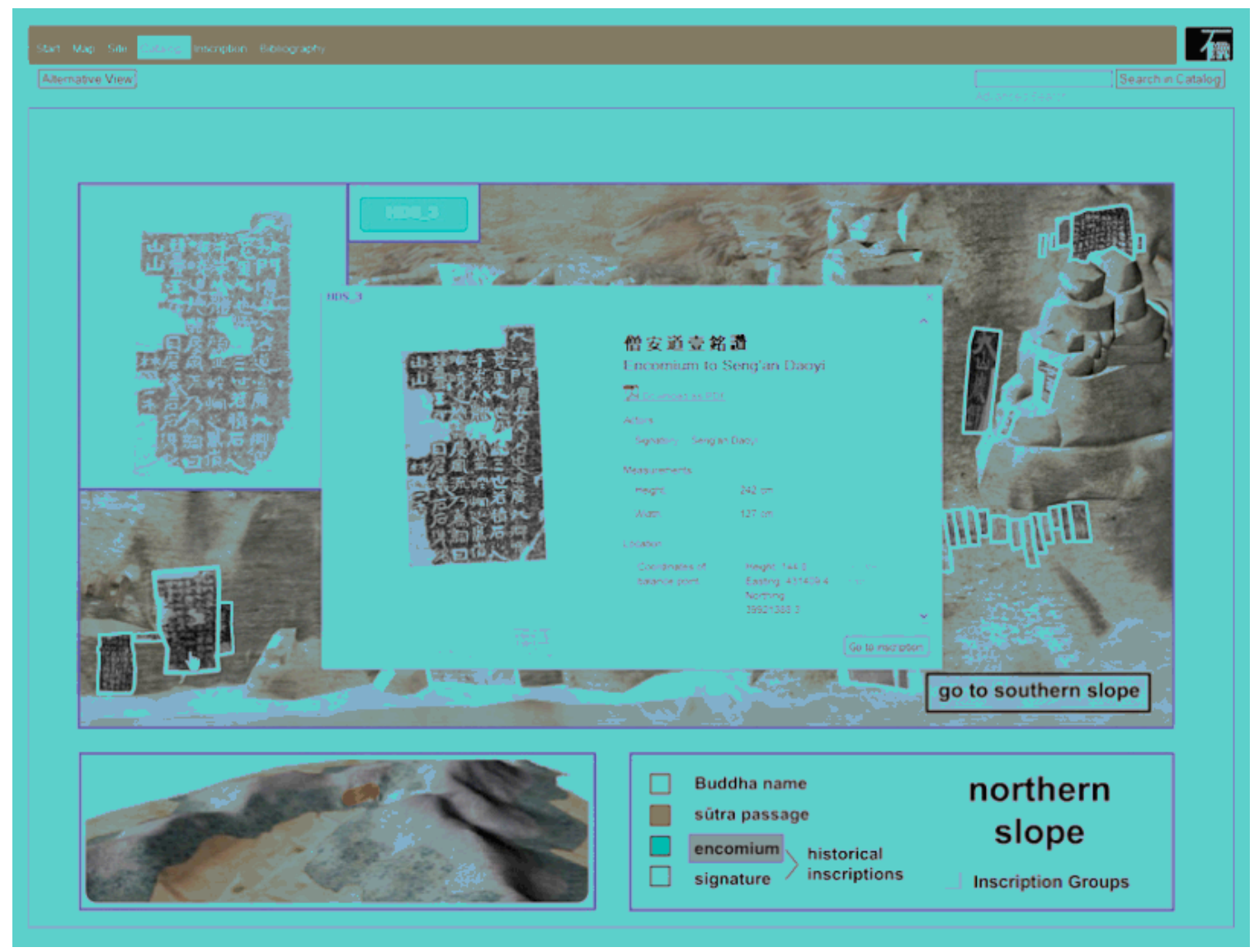

Figure 4 - Catalogue: Flash interface for Mount Hongding

\section{Catalogue}

To access a specific text one needs to open the "Catalogue" of inscriptions. By default, an interactive Flash-navigation shows the site in a rendering from the $3 \mathrm{D}$ model reconstruction. The rock surfaces were photogrammetrically measured by the GeoInformatics team who also constructed 3D models of the sites. Within the model photographs of the rubbings are so exactly positioned, that all individual characters can be 
identified by their coordinates within the global coordinate system (orthophotographs). [11]

Within the Flash-tool users can virtually visit the site, get an impression of the setting, and an overview of the inscriptions. They can load further metadata, like detailed information on the inscriptions measurements, location, and condition (catalogue data). They can also follow cross-links to related inscriptions, or have a closer look at references to academic research, annotated links to available transcriptions, illustrations, or discussions of this specific inscription. Users without the Flash plug-in installed may use the "Alternative View" where all inscriptions are displayed in an interactive list.

\section{Inscription}

Displaying the inscription itself is not as trivial as one might think. This is because there are different layers of information that we believe are necessary to be displayed in one interface. The result is what we call the "Five Layers" model.

- We need to display the rubbing. However, inscriptions can be so huge that characters would not be legible on the screen. We therefore developed a technology that allows to zoom into the rubbing without affecting the size of the images users need to download. By default, almost half of the screen is used for displaying the rubbing.

- We need to display our transcription, or reading, of the text. We call this the "layout text" as characters are arranged in the same way as on the rock surface, i.e. mostly in vertical lines reading from top-right to bottom-left, keeping the original linebrakes. As web-browsers still do not support vertical arrangement of text, characters need to be placed individually by stylesheets.

- Furthermore, we display our English translation of the text. It is shown as continuous text in paragraphs added according to its content, with no regard to linebrakes in the original Chinese layout. 


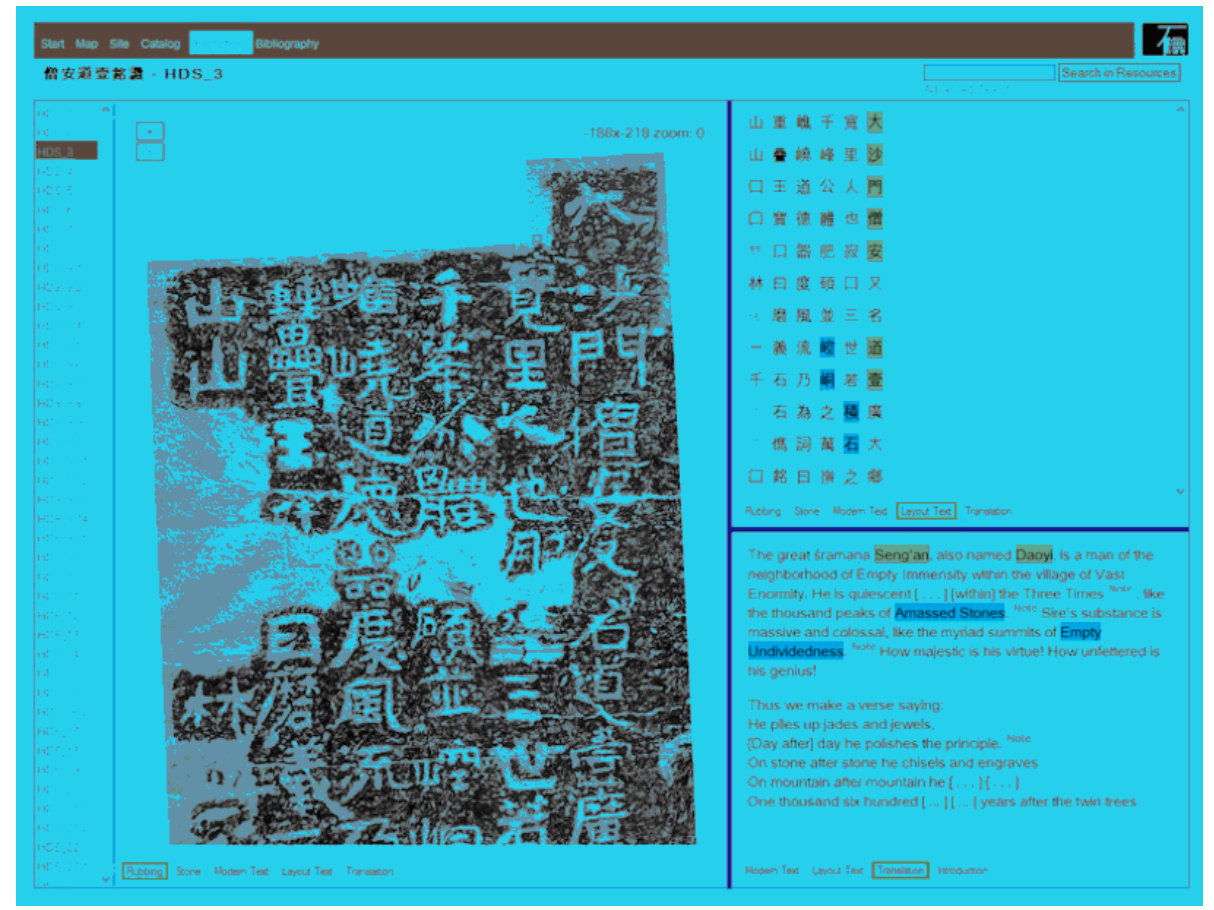

Figure 5. Inscription no.3 from Mount Hongding

- These three segments constitute the default set of information offered for each inscription and constitute an important part of the project's research efforts. But other layers also need to be included.

- In addition to the vertical display of the transcription we also show a "Modern text" version of the inscription, where punctuation is provided and the text can be read horizontally, from left to right.

- To complete the picture we include a "Stone" section providing views of the inscription in-situ, photographs of details, or display the special relation of the text to other inscriptions.

The five layers, or variants of displaying the very same inscription from different points of view, are all incorporated into a single webpage by using a flexible "split-screen" interface and additional buttons within each segment. Users are free to switch content and size of the segments according to their needs. Later on in the project another segment was added to allow for in-site navigation.

\section{Critical apparatus}

Although some inscriptions and even complete sites were only discovered in recent years, many scholars have been puiblishing on the subject, and because of the sometimes difficult conditions (i.e. weathering or general state of preservation) different readings and interpretations of inscriptions exist. We therefore included all information about different readings or textual interpretations in our database, added data about the physical 
condition or the degree of damage of individual characters, and inserted notes and annotations wherever necessary. Visualizing all different kinds of annotations is another challenge for the interface and not all problems are solved yet. The main types of annotation are:

- Information on reading variants. Indicated by red foreground color, the actual data is displayed on mouse-over, like a tooltip.

- Information on character damage. Completely illegible characters are tagged as "unclear" and displayed a squares. In cases where the text is known and missing characters could be supplemented, they are displayed in a light-grey foreground color. c) Indication of names, dates and locations. Wherever possible, we tagged this information in XML. The web-interface uses different background colors for highlighting, like shades of green for names, and blue for locations. Dates are not yet emphasized.

- Technical terms. For a number of inscriptions special Buddhist terms were tagged. They are marked with a dark-grey background color in the "Modern text" segment.

- Notes and references. Annotations on the identification of characters (in the "Modern Text" section), or on translation problems (in the "Translation" section) are marked as "Notes". When the user clicks on them, a small window containing the note opens.

Our visualization of annotations, especially for the critical apparatus is still experimental. Main problems are the many different types of information on the one hand and technical limitations or layout restrictions on the other. We would be very happy to learn from other projects or enter a discussion about possible solutions.

\section{Further development}

One big disadvantage in the current interface is the missing link between a single character in the transcribed text and its representation (i.e. position) on the rubbing. Especially in lager inscriptions, parallel reading of rubbing and text with optional switching between both would be highly desirable. To achieve this, a project was started in 2007 that will close this gap [12]. We have already produced a tool that allows the slicing of inscriptions on a character base and produces coordinates for each slice that relate to both, the position on the rubbing and the Unicode code-point of the transcribed character. The characters database will contain the images of all identified characters from the Shandong inscriptions on rubbings and stone, including variants and divergent readings. Hopefully, this tool will become helpful for answering questions relevant to arthistory, like if there is a relation between the style of the calligraphy and the content of the inscription. But this will be the topic of another paper. 


\section{ACKNOWLEDGEMENTS}

This paper was supported by the Chiang Ching-kuo Foundation for International Scholarly Exchange.

\section{References}

[1] Standard reference for Buddhist texts is the corpus digitized by the Chinese Buddhist Electronic Text Association (CBETA), http://www.cbeta.org

[2] http://www.w3.org/XML/

[3] TEI: Text Encoding Initiative; Guidelines: http://www.tei-c.org/Guidelines/P5/

[4] http://www.loc.gov/standards/mods/

[5] http://www.loc.gov/standards/mads/

[6] http://exist-db.org/

[7] http://www.oxygenxml.com/

[8] The site now requires a password, please contact us at info@stonesutras.org for more information.

[9] http://geoserver.org

[10] http://code.google.com/apis/earth/

[11] The rubbings are geometrically corrected according to the surface topography, i.e. "orthorectified", resulting in orthophotographs, or rather "orthorubbings".

[12] The project called "Visual Data bank of Sutras Engraved on Rocks in Shandong Province" is generously supported by the Chiang Ching-kuo Foundation for International Scholarly Exchange. 